\title{
A bifunctional nucleoside probe for the inhibition of the human immunodeficiency virus-type 1 reverse transcriptase
}

Tyler A. Shaw ${ }^{1}$, Christopher J. Ablenas', Geneviève F. Desrochers ${ }^{1}$, Megan H. Powdrill ${ }^{1}$, Didier A. Bilodeau', Jean-François Vincent-Rocan', Meijuan Niu², Anne Monette², Andrew J. Mouland ${ }^{2,3}$, André M. Beauchemin ${ }^{1}$ and John Paul Pezacki ${ }^{1 *}$

'Department of Chemistry and Biomolecular Science, University of Ottawa, 1o Marie Curie, Ottawa, Ontario K1N 6N5, Canada; ${ }^{2} L a d y$ Davis Institute at the Jewish General Hospital, 3755 Chemin de la Côte-SainteCatherine, Montréal, QC H3T 1E2, Canada; 3Department of Medicine, McGill University, 3999 Côte-SteCatherine Road, Montréal, Québec H3T 1E2, Canada

\section{Supplementary Tables}

Table S1. Percentage coverage of HIV RT with chemically modified peptides by LC-MS/MS.

\begin{tabular}{lccc|ccc} 
& \multicolumn{3}{c}{ p66 } & \multicolumn{3}{c}{$\mathrm{p} 51$} \\
\cline { 2 - 7 } & Labelled & Peptide ID ctrl & Labelling ctrl & \multicolumn{2}{|c}{ Labelled Peptide ID ctrl Labelling ctrl } \\
\hline Probe 1 & 86.0 & 84.8 & 86.2 & 98.4 & 96.6 & 99.3 \\
\hline Probe 2 & 87.1 & 87.1 & 85.2 & 92.4 & 91.7 & 91.7 \\
\hline Probe 3 & 88.3 & 86.7 & 87.1 & 98.7 & 95.7 & 92.6 \\
\hline
\end{tabular}

Table S2. LC-MS/MS hits of p66 subunit peptides labeled with azauracil probes.

\begin{tabular}{|c|c|c|c|c|c|c|c|c|c|}
\hline Probe & Annotated Sequence & Modifications & $\begin{array}{l}\text { Missed } \\
\text { Cleavages }\end{array}$ & Charge & $\mathbf{m} / \mathbf{z}[\mathrm{Da}]$ & $\mathrm{MH}+[\mathrm{Da}]$ & DeltaM [ppm] & RT [min] & XCorr \\
\hline & [R].GsELPIsPIETVPVK.[L] & S3 & 0 & 3 & 623.3219 & 1867.9511 & 6.769469224 & 39.71513 & 1.3253503 \\
\hline \multirow[t]{2}{*}{1} & [K].yARMRGAHtNDVK.[Q] & Y354, T362 & 2 & 3 & 607.61139 & 1820.8196 & 0.115003425 & 43.6116 & 1.0448167 \\
\hline & [R].WGLTtPDKK.[H] & T216 & 1 & 2 & 605.80383 & 1210.6004 & -6.015098489 & 35.29723 & 1.0266793 \\
\hline \multirow[t]{3}{*}{2} & [K].HQKEPPFLWmGyELHPDKWTVQPIVLPEK.[D] & Y232 & 2 & 6 & 621.48138 & 3723.8519 & -3.30851372 & 44.89825 & 1.5395731 \\
\hline & [-].mRGsELPIsPIETVPVKLKPGmDGPK.[V] & S3 & 2 & 5 & 650.93835 & 3250.6627 & 7.034926156 & 50.85798 & 1.0219656 \\
\hline & [-].mRGSELPIsPIEtVPVKLKPGmDGPK.[V] & $\mathrm{S} 3, \mathrm{~T} 7$ & 2 & 5 & 650.93835 & 3250.6627 & 7.034926156 & 50.85798 & 1.0607207 \\
\hline 3 & [R].GTKALtEVIPLTEEAELELAENR.[E] & T290 & 1 & 3 & 916.46368 & 2747.3765 & -8.458603087 & 56.65395 & 1.8054019 \\
\hline
\end{tabular}




\section{Supporting Information}

Table S3. LC-MS/MS hits of $\mathbf{p} 51$ subunit peptides labeled with azauracil probes.

\begin{tabular}{|c|c|c|c|c|c|c|c|c|c|}
\hline Probe & Annotated Sequence & Modifications & $\begin{array}{l}\text { Missed } \\
\text { Cleavages }\end{array}$ & Charge & $\mathbf{m} / \mathbf{z}[\mathrm{Da}]$ & $\mathrm{MH}+[\mathrm{Da}]$ & DeltaM [ppm] & $\mathbf{R T}$ [min] & XCorr \\
\hline 2 & [K].SVTVLDVGDAyFSVPLDEDFRK.[Y] & Y115 & 1 & 4 & 660.07355 & 2637.2724 & 1.161668674 & 52.10741 & 1.015239 \\
\hline & [K].IGPENPyNtPVFAIKKK.[D] & Y56, T58 & 2 & 4 & 590.30676 & 2358.2052 & 0.513781354 & 42.7129 & 1.1230226 \\
\hline 3 & [K].IGPENPyNtPVFAIKKK.[D] & Y56, T58 & 2 & 4 & 590.30713 & 2358.2067 & 1.134950576 & 41.95076 & 1.0856485 \\
\hline
\end{tabular}

\section{Supplementary Materials, Methods and Figures}

The [3+2] copper-catalyzed azide-alkyne cycloaddition (CuAAC/Click) reaction of AzTTP/AzT with probe 3. The click reaction was prepared in $1 \mathrm{X}$ PBS using $10 \mathrm{mM}$ Ascorbate, $10 \mathrm{mM} \mathrm{CuSO}_{4}$, 1mM TBTA, $11 \mathrm{mM}$ probe 3, and $10 \mathrm{mM}$ AzTTP or AzT. The components were reacted for 1 hour and excess copper was removed using Nickel NTA resin. The resin was washed with PBS, stripped three times with Ni2+ stripping buffer (20 $\mathrm{mM} \mathrm{NaHPO}_{4} \mathrm{pH}$ $7.4,500 \mathrm{mM} \mathrm{NaCl}, 50 \mathrm{mM}$ EDTA) and dried before adding the click reaction and collecting the reaction by centrifuging at $700 \mathrm{x} g$ for 2 minutes. AzTTP (Cedarlane) and AzT (Sigma Aldrich) were purchased from commercial sources. Spectral data for probe 3 matched previously reported characterization data. ${ }^{1}$ After quantitative coupling via $[3+2]$ CuAAC, the product (approximately $0.8 \mathrm{mg}$ of 4 and $1.2 \mathrm{mg}$ of ${ }_{5}$ ) was characterized by ${ }^{1} \mathrm{H}-\mathrm{NMR},{ }^{13} \mathrm{C}-\mathrm{NMR}$, ${ }^{3} \mathrm{P}-\mathrm{NMR}$, and HMBC for 4, and ESI mass spectrometry, ${ }^{1} \mathrm{H}-\mathrm{NMR},{ }^{13} \mathrm{C}-\mathrm{NMR}$, and HSQC for $\mathbf{5}$ (ESI spectra were measured from $\mathrm{D}_{2} \mathrm{O}$ samples), supporting structural assignment. Spectral data for the products was also consistent with similar previously reported AzT "clicked" analogues. ${ }^{2}$<smiles>Cc1cn([C@H]2C[C@H](n3cc(Cn4c(=O)[nH]nc(C5CCCO5)c4=O)nn3)[C@@H](COP(=O)(O)OP(=O)(O)OP(=O)(O)O)O2)c(=O)[nH]c1=O</smiles>

$\mathrm{C}_{20} \mathrm{H}_{27} \mathrm{~N}_{8} \mathrm{O}_{16} \mathrm{P}_{3}$ ${ }^{1} \mathrm{H}-\mathrm{NMR}\left(600 \mathrm{MHz}, \mathrm{D}_{2} \mathrm{O}\right) \delta 8.20(\mathrm{~s}, 1 \mathrm{H}), 7.89(\mathrm{~s}, 1 \mathrm{H}), 6.60(\mathrm{t}, J=7.3 \mathrm{~Hz}, 1 \mathrm{H}), 5.64(\mathrm{dt}, J=7.1,3.2 \mathrm{~Hz}, 1 \mathrm{H}), 5.18(\mathrm{~s}$, $2 \mathrm{H}), 5.08-5.00(\mathrm{~m}, 1 \mathrm{H}), 4.66-4.61(\mathrm{~m}, 1 \mathrm{H}), 4.35-4.24(\mathrm{~m}, 2 \mathrm{H}), 3.99(\mathrm{q}, J=7.2 \mathrm{~Hz}, 1 \mathrm{H}), 3.92-3.87(\mathrm{~m}, 1 \mathrm{H}), 2.83-$ $2.75(\mathrm{~m}, 2 \mathrm{H}), 2.33-2.26(\mathrm{~m}, 1 \mathrm{H}), 2.09-2.03(\mathrm{~m}, 1 \mathrm{H}), 2.03-1.97(\mathrm{~m}, 2 \mathrm{H}), 1.95(\mathrm{~s}, 3 \mathrm{H}) .{ }^{1{ }^{13}}{ }^{1}-\mathrm{NMR}\left(151 \mathrm{MHz}, \mathrm{D}_{2} \mathrm{O}\right) \delta$ $166.61,156.35,150.19,141.59,137.23,111.98,85.57,75.55,69.07,65.83,61.20,59.65,37.45,34.75,29.23,24.86,11.67$. HMBC ${ }^{1} \mathrm{H}-\mathrm{NMR}\left(6 \mathrm{oo} \mathrm{MHz}, \mathrm{D}_{2} \mathrm{O}\right) \delta 8.15,8.14,7.85,7.84,7.84,6.55,6.55,5.13,5.13,5.13,5.13,3.95,3.94,3.94,3.85$, $3.85,3.84,2.75,2.74,2.50,2.25,2.25,2.01,2.01,2.01,2.00,2.00,1.96,1.95,1.95,1.90,1.90,1.90,1.79$. HMBC ${ }^{13}$ C-NMR 
(151 MHz, D $\mathrm{O}) \delta 141.59,141.64,166.69,11.68,151.73,137.15,151.72,141.61,156.36,125.10,150.47,75.44,24.72,29.28$, 24.71, 75.53, 29.26, 85.23, 61.05, 61.22, 24.93, 145.02, 69.01, 145.02, 75.54, 11.67, 24.86, 69.04, 75.75, 29.25, 166.66, 137.26, 111.98, 11.68. ${ }^{31} \mathrm{P}$ NMR (203 MHz, $\left.\mathrm{D}_{2} \mathrm{O}\right) \delta-9.26(\mathrm{br}, 1 \mathrm{P}),-11.73(\mathrm{~d}, J=20.1 \mathrm{~Hz}, 1 \mathrm{P}),-22.82(\mathrm{t}, J=19.3 \mathrm{~Hz}, 1 \mathrm{P})$.

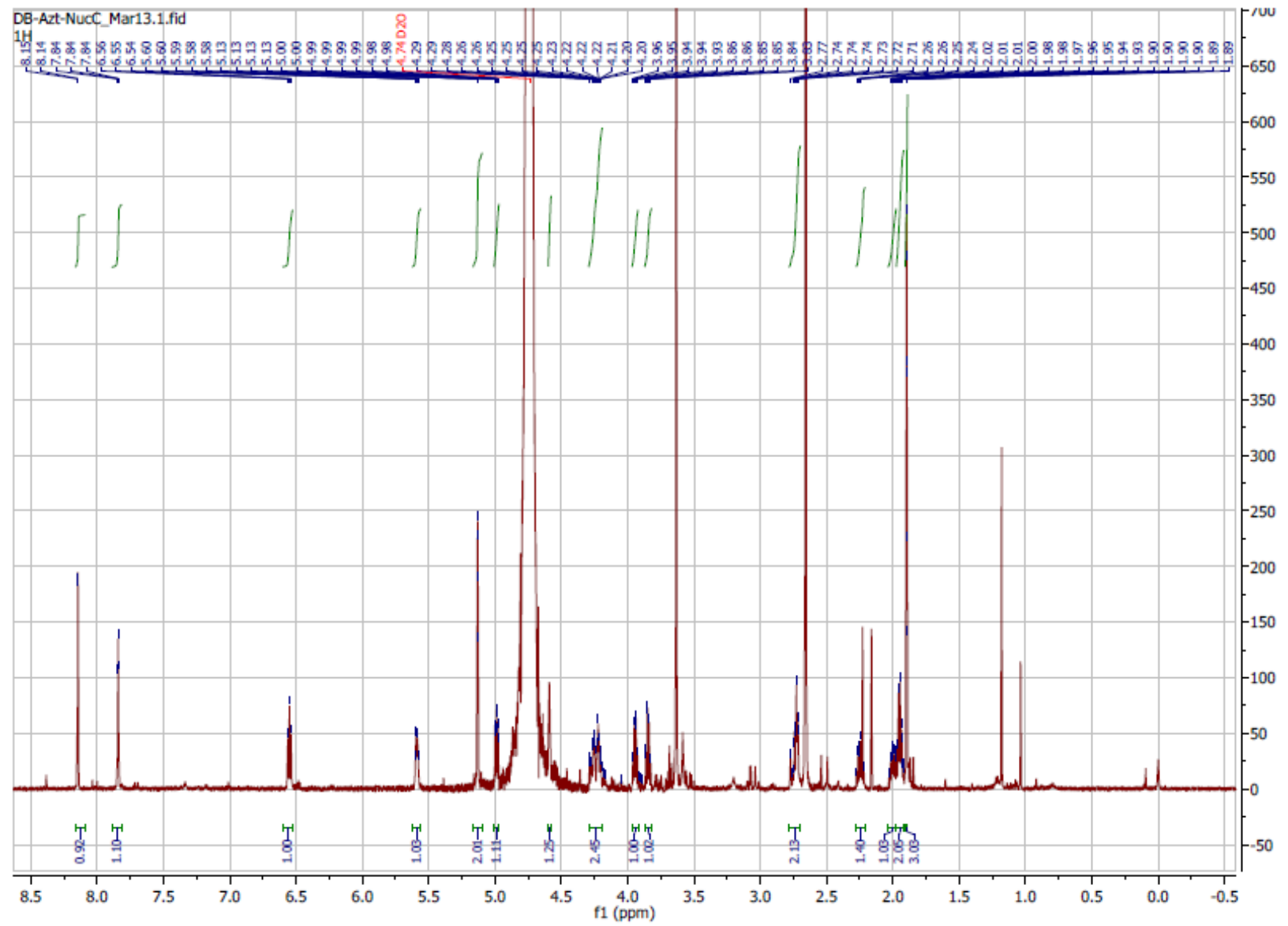

Figure S1. ${ }^{1} \mathrm{H}-\mathrm{NMR}$ spectrum of Azauracil-AzTTP. 
Supporting Information

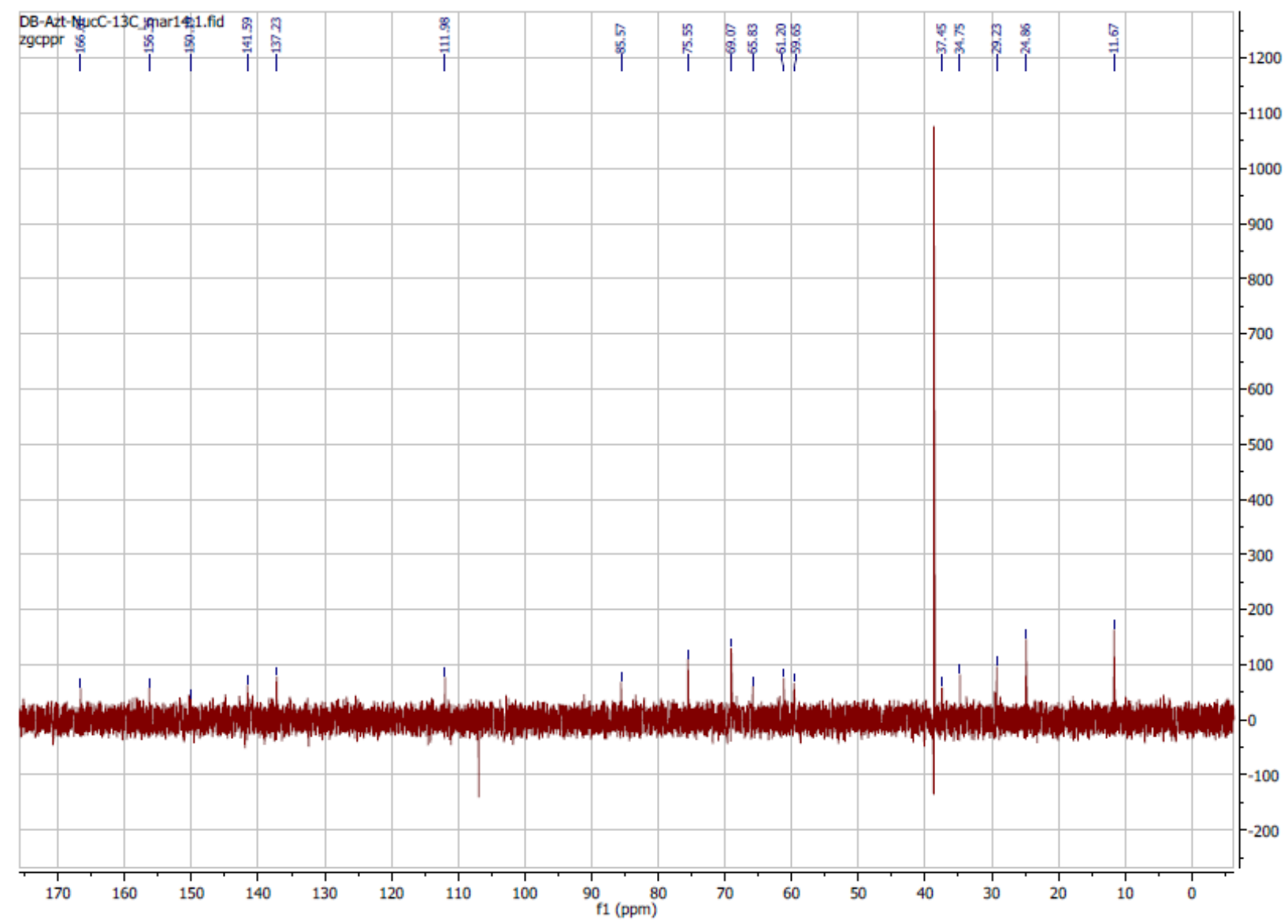

Figure S2. ${ }^{13}$ C-NMR spectrum of Azauracil-AzTTP. 


\section{Supporting Information}

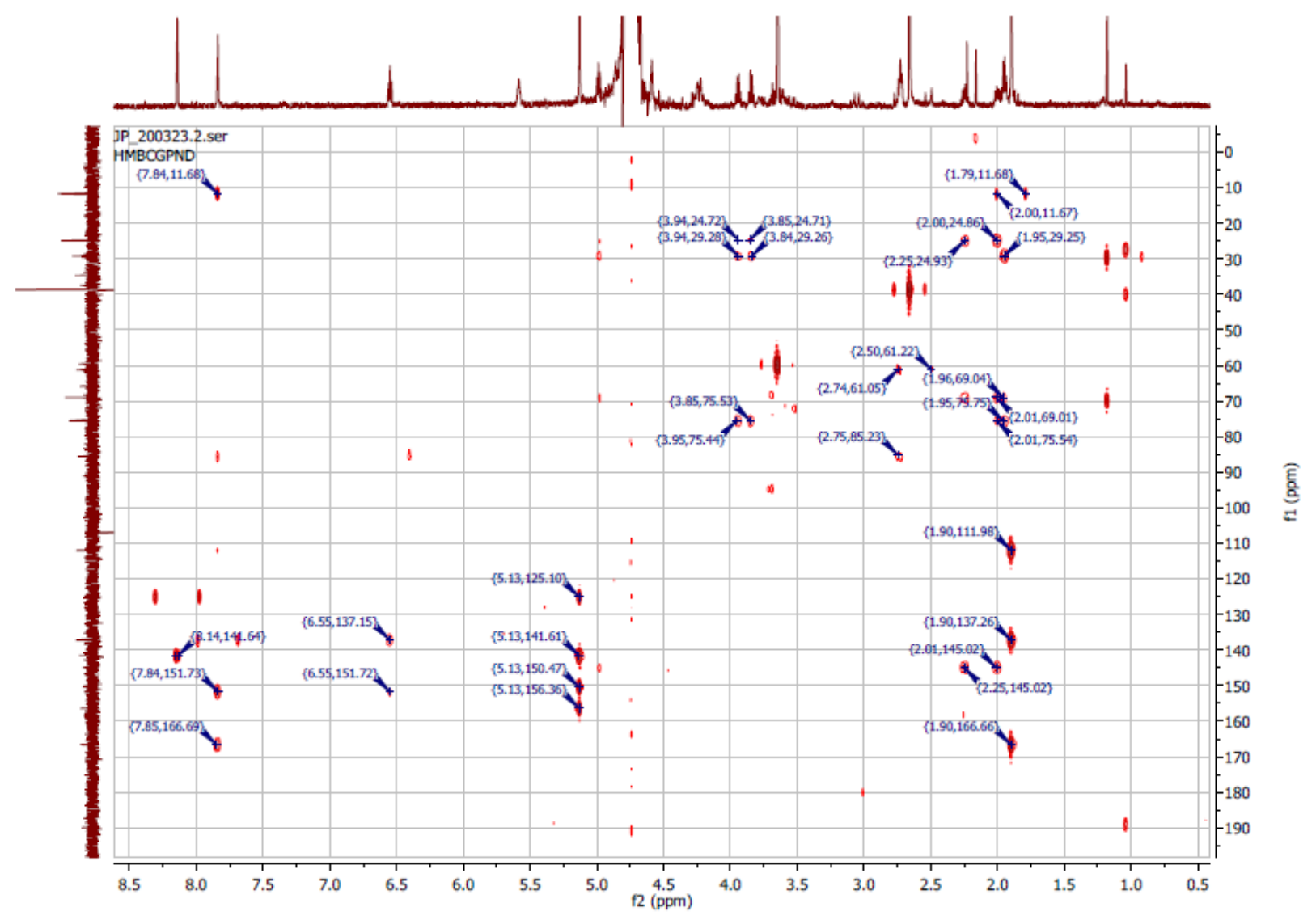

Figure S3. HMBC spectrum of Azauracil-AzTTP. 


\section{Supporting Information}

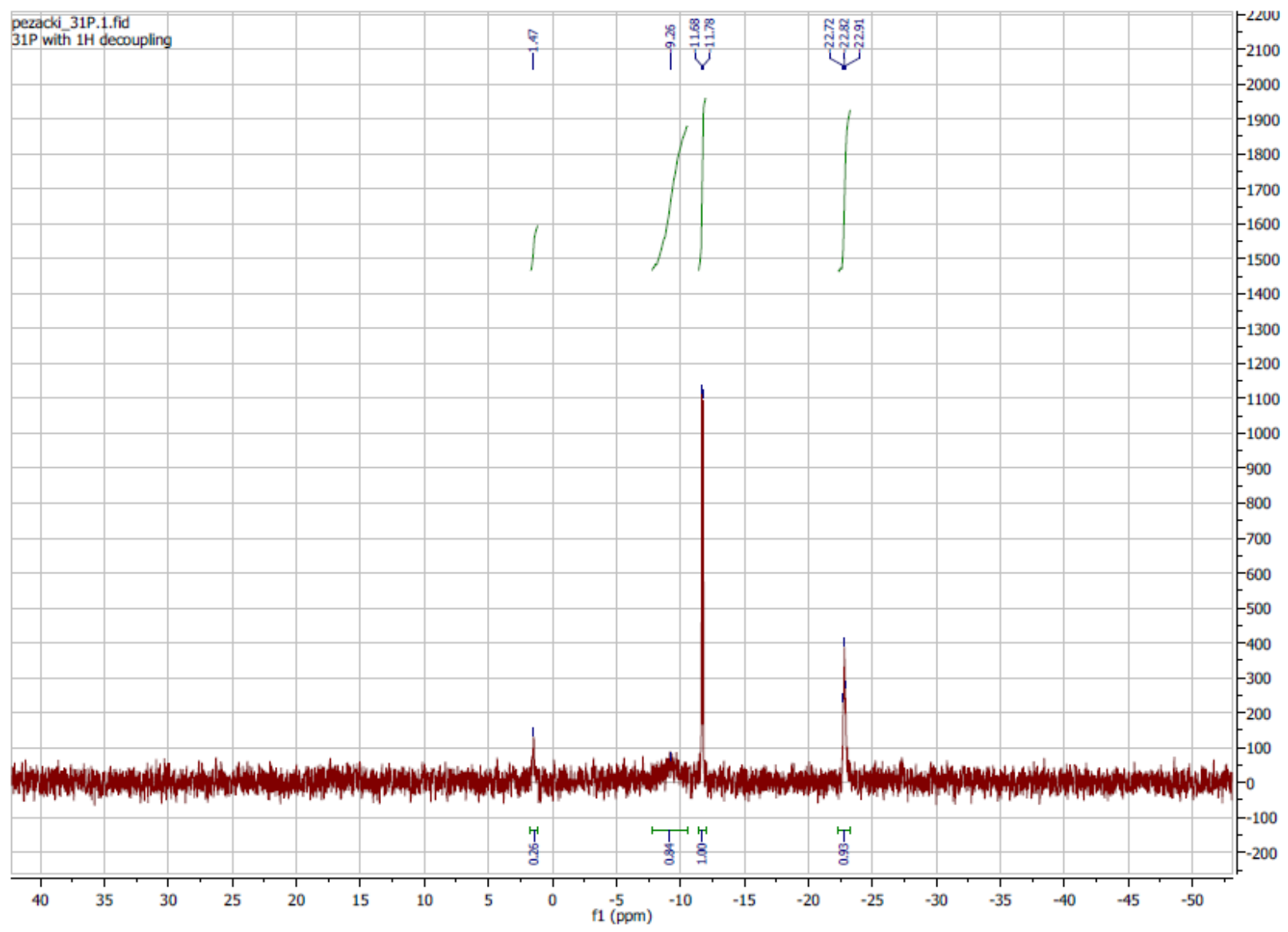

Figure S4. ${ }^{31} \mathrm{P}$ ( ${ }^{1} \mathrm{H}$ decoupled) spectrum of Azauracil-AzTTP. 


\section{Supporting Information}

\section{Azauracil-AzT (5)}<smiles>Cc1cn([C@H]2C[C@H](n3cc(CN4CC(C5CCCO5)=NNC4=O)nn3)[C@@H](CO)O2)c(=O)[nH]c1=O</smiles>

HRMS (ESI+) [M+Na]: calcd. for $\mathrm{C}_{20} \mathrm{H}_{23} \mathrm{D}_{3} \mathrm{~N}_{8} \mathrm{O}_{6} \mathrm{Na}^{+}:$514.1848, found: 514.2145

${ }^{1} \mathrm{H}-\mathrm{NMR}\left(600 \mathrm{MHz}, \mathrm{D}_{2} \mathrm{O}\right) \delta 8.10(\mathrm{~s}, 1 \mathrm{H}), 7.68(\mathrm{~s}, 1 \mathrm{H}), 6.39(\mathrm{t}, J=6.5 \mathrm{~Hz}, 1 \mathrm{H}), 5.36(\mathrm{dt}, J=8.9,6.1 \mathrm{~Hz}, 1 \mathrm{H}), 5.13(\mathrm{~s}$, $2 \mathrm{H}), 4.97-5.00(\mathrm{~m}, 1 \mathrm{H}), 4.40(\mathrm{dt}, J=6.7,3.8 \mathrm{~Hz}, 1 \mathrm{H}), 3.94(\mathrm{q}, J=7.0 \mathrm{~Hz}, 1 \mathrm{H}), 3.83-3.87(\mathrm{~m}, 2 \mathrm{H}), 3.74(\mathrm{dd}, J=12.7$, $4.4 \mathrm{~Hz}, 1 \mathrm{H}), 2.87-2.93(\mathrm{~m}, 1 \mathrm{H}), 2.82-2.73(\mathrm{~m}, 1 \mathrm{H}), 2.29-2.20(\mathrm{~m}, 1 \mathrm{H}), 2.04-1.90(\mathrm{~m}, 3 \mathrm{H}), 1.86\left(\mathrm{~s},{ }_{3} \mathrm{H}\right) .{ }^{13} \mathrm{C}-\mathrm{NMR}$ $\left(151 \mathrm{MHz}, \mathrm{D}_{2} \mathrm{O}\right) \delta 166.57,156.36,151.60,150.39,144.92,137.78,124.39,111.49,85.51,84.18,75.55,69.08,60.47,59.55$, $38.70,36.84,34.73,29.16,24.88,11.53 .{ }^{13} \mathrm{C} / \mathrm{HSQC}$ NMR $\left(151 \mathrm{MHz}, \mathrm{D}_{2} \mathrm{O}\right) \delta 124.51,137.77,85.54,59.56,34.72,75.56$, 84.21, 69.05, 69.08, 60.47, 60.47, 36.85, 36.83, 29.09, 29.08, 24.87, 11.47 ${ }^{1} \mathrm{H} / \mathrm{HSQC}$ NMR (6oo MHz, $\left.\mathrm{D}_{2} \mathrm{O}\right) \delta 8.09$, $7.68,6.39,5.36,5.13,4.98,4.40,3.94,3.85,3.84,3.74,2.90,2.78,2.25,2.01,1.95,1.86$.

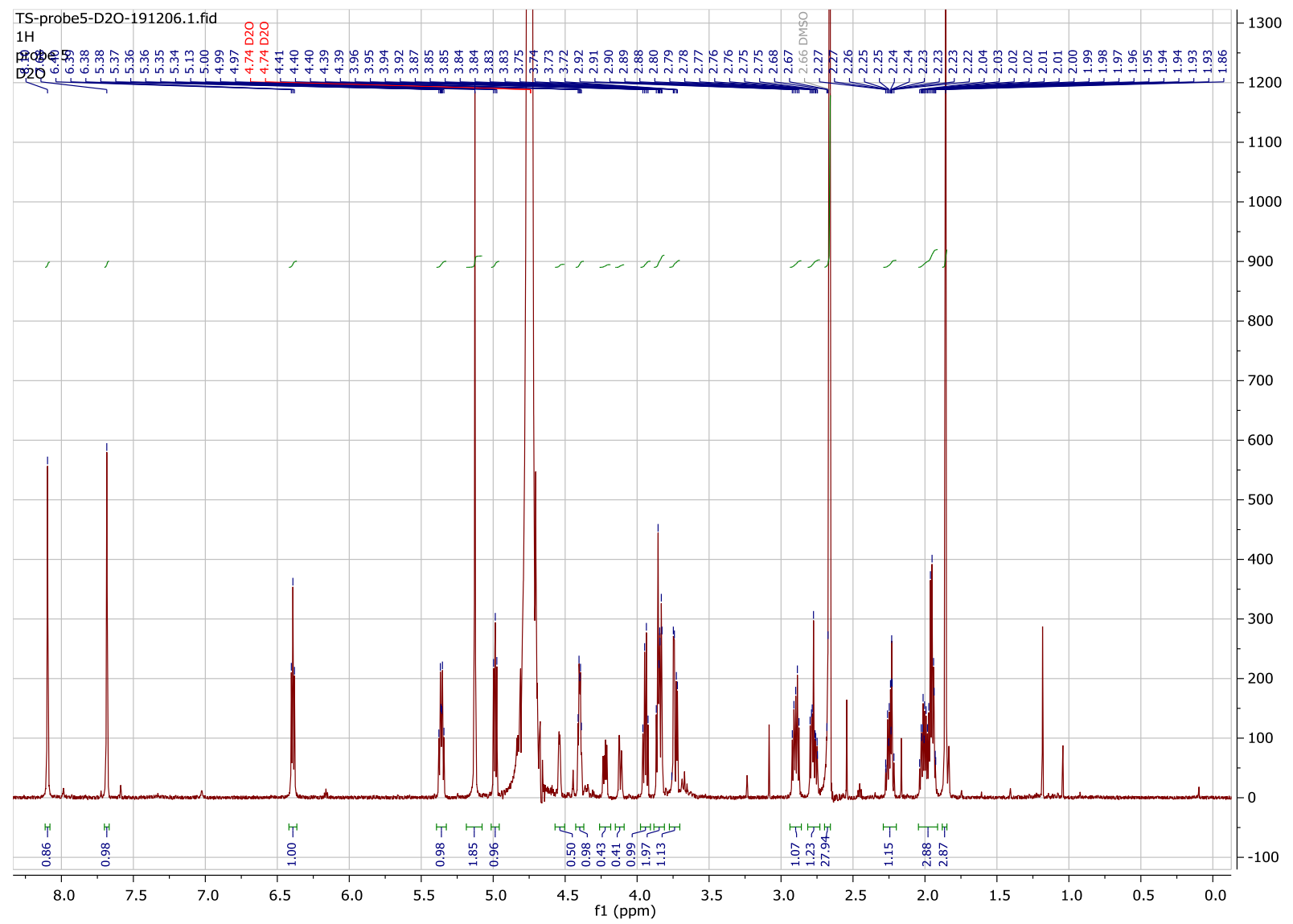

Figure S5. $^{1} \mathrm{H}-\mathrm{NMR}$ spectrum of Azauracil-AzT. 


\section{Supporting Information}

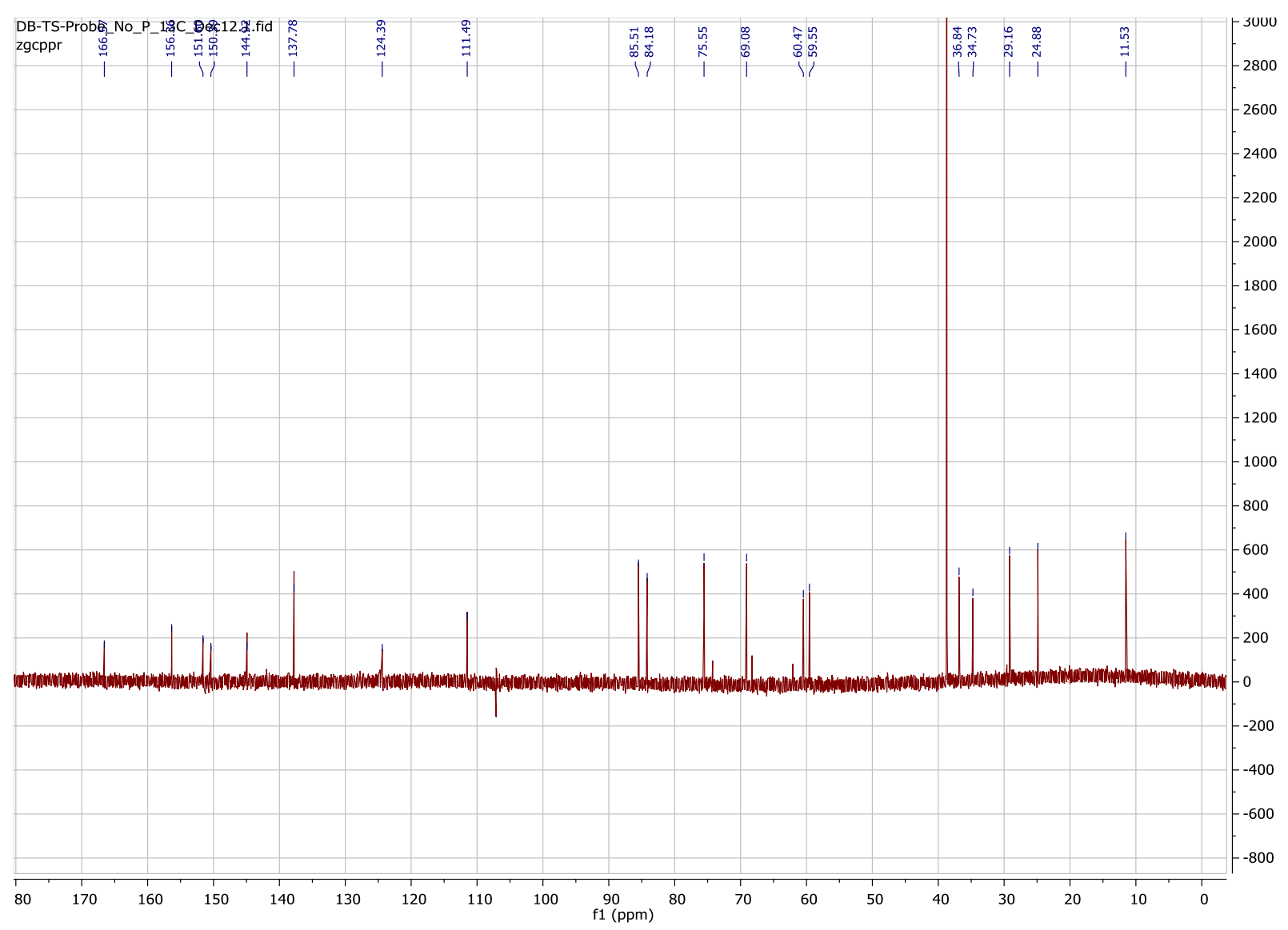

Figure S6. ${ }^{13} \mathrm{C}-\mathrm{NMR}$ spectrum of Azauracil-AzT. 


\section{Supporting Information}

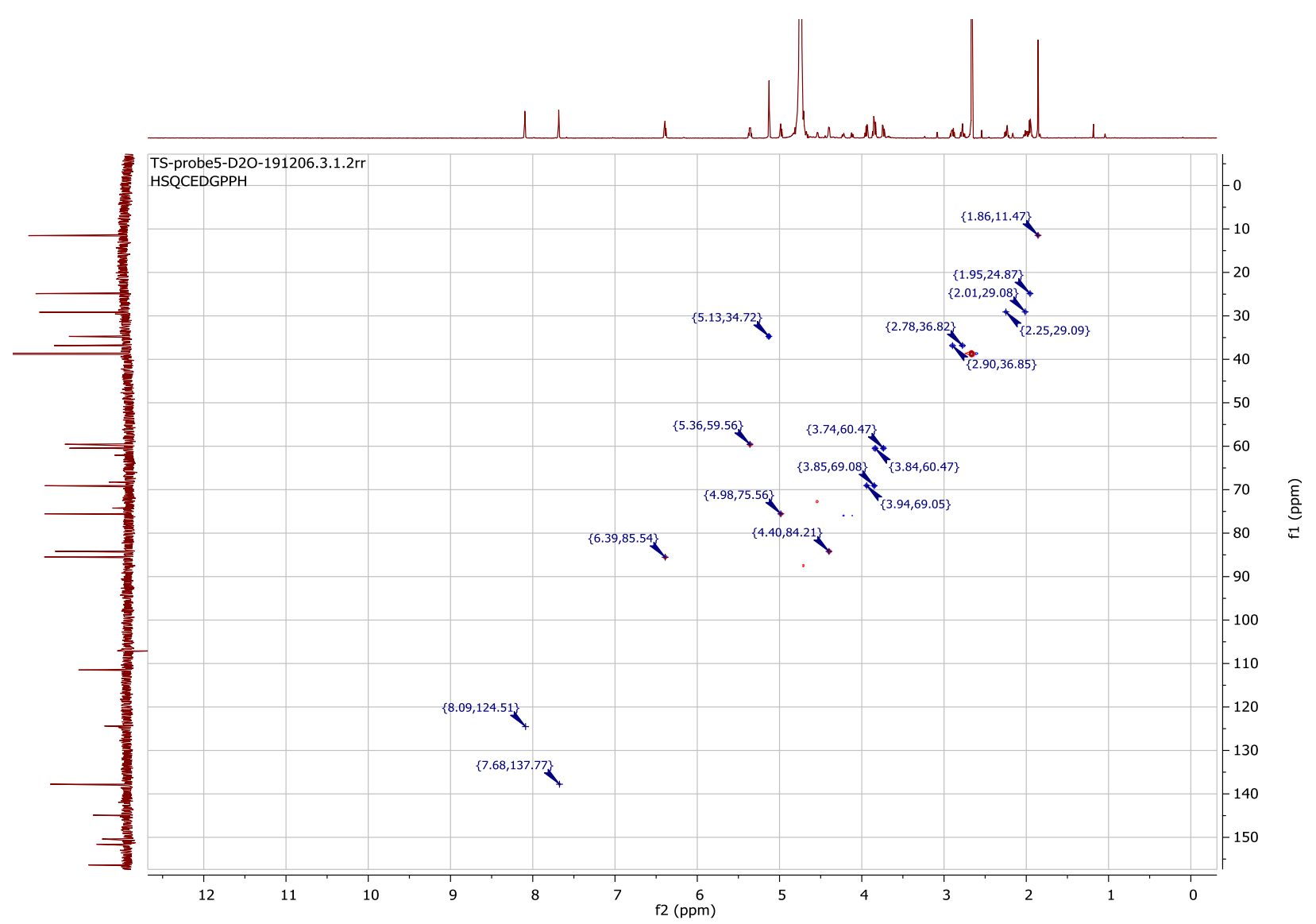

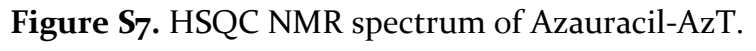

\section{Supplementary References}

1. J.-F. Vincent-Rocan, R. A. Ivanovich, C. Clavette, K. Leckett, J. Bejjani and A. M. Beauchemin, Chem. Sci., 2016, 7, 315-328.

2. L. Zhou, A. Amer, M. Korn, R. Burda, J. Balzarini, E. De Clercq, E. R. Kern and P. F. Torrence, Antivir. Chem. Chemother., 2005, 16, 375-383. 